\title{
Breast Tumour Identification based on Chimera Overset Grid with Spline Interpolation in Forward-Backward Time-Stepping
}

\author{
B.S.Wee ${ }^{1,2}$, K.A.H.Ping ${ }^{1 *}$ and S.Sahrani ${ }^{1}$ \\ ${ }^{1}$ Applied Electromagnetic Research Group, Department of Electrical \& Electronic Engineering, Faculty of Engineering, \\ Universiti Malaysia Sarawak, 94300 Kota Samarahan, Sarawak, Malaysia \\ ${ }^{2}$ Department of Electrical Engineering, Politeknik Mukah, 96400 Mukah, Sarawak, Malaysia
}

\begin{abstract}
Active microwave imaging (MI) technique has been recently recommended as a new technology for early detection of malignant breast tumours. Finite-difference Time-domain (FDTD) method is an effective numerical tool for computational electrodynamics modelling. However, the main drawback of the FDTD method is difficult to model the curved boundaries and small features of arbitrary shaped fibroglandular region due to its restriction to inherent orthogonal grids. To address this issue, Chimera Overset Grid (COG) method with spline interpolation in Forward-Backward TimeStepping (FBTS) inverse scattering technique was proposed to overcome the limitation of FDTD method. In order to analyse the accuracy of this new method to detect a malignancy tumour embedded in different classes of breast parenchymal density, the homogeneous mostly fatty breast and extremely dense breast were chosen as Case A, and Case B, respectively. The Mean Squared Error (MSE) and normalised cost error functional for reconstructed dielectric profiles utilising the proposed method achieved significantly lower values than the FDTD method in FBTS inverse scattering. The results indicated that the proposed method could accurately identify malignancy tumour location and reconstruct the breast composition in dielectric profiles.
\end{abstract}

Keywords: Chimera overset grid; biquadratic spline interpolation; breast tumour; inverse scattering

\section{INTRODUCTION}

Breast cancer is the most common cancer amongst women that causes death (Siegel et al., 2019). In 2019, the American Cancer Society reported that the new breast cancer cases are approximately $1,762,450$ and 606,880 women will die from this disease (American Cancer Society, 2019). Early detection and diagnosis of breast cancer with the appropriate treatment is the key to recovery. Therefore, annual breast examination is important for early detection of malignant tumours. There are several types of breast screening techniques, such as Xray mammography, breast ultrasound, Magnetic Resonance Imaging (MRI) and Scintimammography. X-ray mammography is currently an important and efficient technique tool by which to detect, diagnose and evaluate breast cancer (Radiological Society of North America Inc., 2019). However, this technique has several limitations due to difficulties in detecting breast tumours in their earlier stages especially for the dense breast tissue of young patients (Brennan \& Houssami, 2016). It also suffers from a relatively high number of misses and false-detection rates (Tartar et al., 2008). It is an uncomfortable and perhaps painful process for patients due to the breast compression involved. Ionising radiations are released during the screening process (Hang et al., 2017).

Therefore, microwave imaging (MI) technology has dreamed of using non-invasive, cost-effective, convenient, and non-ionizing electromagnetic waves to expose the human body for cancers detection (Wang, 2019). The last decade has 
seen significant progress towards this dream by using the MI technology for breast cancer detection (Aldhaeebi et. al., 2020; Li et. al., 2017; Mashal et. al., 2010; Takenaka et. al., 2010; Winter et al., 2009). The rationale for MI is the high dielectric properties contrast between the cancerous and healthy tissues (Gao et al., 2015). FDTD method is a numerical analysis technique used for solving the nonlinear problem in electromagnetic scattering (Schneide, 2016; Yee, 1996). FDTD solutions can cover a wide frequency range without any extra computational work. Therefore, the timedomain inversion method can be used to increase detection and reconstruction of buried objects. However, the main limitation of the FDTD method is difficult to exactly generate meshes for electromagnetic structures with curved boundaries and small features due to its restriction to inherent orthogonal grids (Jiménez-Mejía \& Herrera-Murcia, 2015; Nilavalan et al., 2002). To address this issue, the Chimera Overset Grid (COG) method with biquadratic spline or $\mathrm{B}_{2}$-spline interpolation in Forward-Backward Time Stepping (FBTS) inverse scattering was proposed in this paper. The geometrically complex problem can be greatly simplified by using the OGG-FDTD method with biquadratic spline interpolation. Previously, we proposed this numerical method to measure the EM scattered field around an unknown object in a different medium (Wee et al., 2018). In this paper, OGG-FDTD method with biquadratic spline interpolation is integrated with FBTS inverse scattering for early detection of malignant breast tumour.

The two-dimensional (2D) numerical simulations in transverse magnetic (TM) z-plane are carried out to investigate the interior of non-dispersive breast composition in free space. The breast is composed of four kinds of tissues with dielectric properties: skin, adipose, fibroglandular, and tumour. Broadband microwave signals are utilising in this simulation for reconstructing the quantitative images of breast. The aim of this study is to accurately determine the shape, size, location, internal permittivity and conductivity of healthy and cancerous breast tissues. The remains of the paper are organised as follows. The method for reconstruction the breast composition is clearly explained in Section 2. The simulation results and the study finding are discussed in Section 3. The research conclusion is summarised in Section 4.

\section{METHOD}

\section{A. COG Method with Biquadratic Spline Interpolation}

The Chimera Overset Grid (COG) method, also known as Overset Grid Generation (OGG) method is shown in Figure 1.

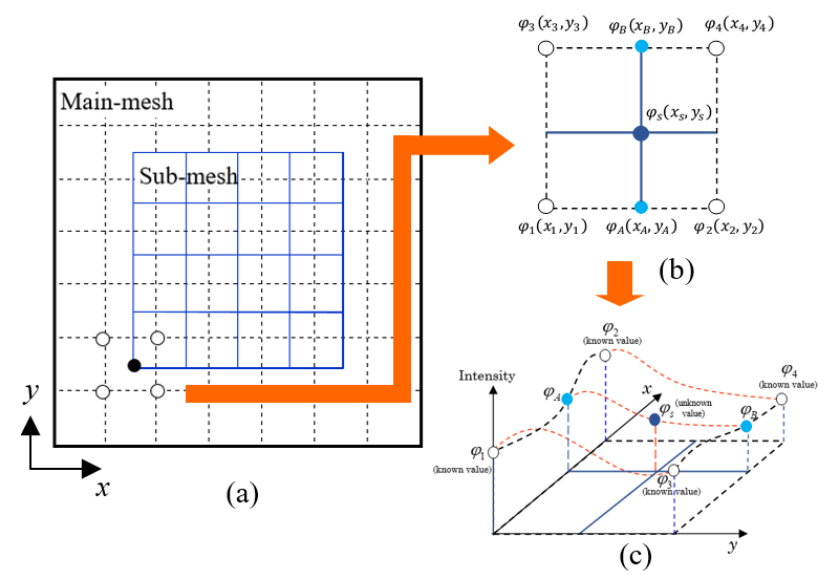

Figure 1. COG scheme: (a) Main-mesh and sub-mesh, (b) Four points biquadratic spline interpolation, (c) Known and unknown values at main-mesh and sub-mesh, respectively

The OGG method consists of two grids that are main-mesh and sub-mesh, as illustrated in Figure 1(a). The main-mesh is also known as FDTD lattice which is generated about a computation domain. The sub-mesh is known as the minor grid which is placed to form a single mesh on top of the mainmesh. The unknown values for the overlapping region can be determined by interpolating technique. Biquadratic spline interpolation technique was proposed in this study due to it can produce high resolution images, efficient interpolation and smooth curve. Figure 1(b) is subtracted from Figure 1(a) as four points of biquadratic spline interpolation technique. The points of $\varphi_{1}, \varphi_{2}, \varphi_{3}$ and $\varphi_{4}$ are the four known values from the main mesh while the point $\varphi_{s}$ is the unknown value from the sub-mesh. Figure 1(c) is a side view of Figure 1(b) at $x-y$ plane with intensity in 2D. The unknown value of $\varphi_{s}$ is interpolated by using the $\varphi_{A}$ and $\varphi_{B}$ at $y$-axis as follows:-

$\varphi_{S}=\left[\frac{d_{j+1}-d_{j}}{2\left(y_{B}-y_{A}\right)}\right]\left(y_{S}-y_{A}\right)^{2}+d_{j}\left(y_{S}-y_{A}\right)+\varphi_{A}$

$$
j=0,1,2, \ldots, m
$$

where,

$$
d_{j}=0, d_{j+1}=\frac{2\left(\varphi_{B}-\varphi_{A}\right)}{y_{B}-y_{A}}-d_{j}, j=0,1,2, \ldots, m
$$




$$
\begin{gathered}
\varphi_{A}=\left[\frac{d_{i+1}-d_{i}}{2\left(x_{2}-x_{1}\right)}\right]\left(x_{A}-x_{1}\right)^{2}+d_{i}\left(x_{A}-x_{1}\right)+\varphi_{1} \\
\because d_{i}=0, d_{i+1}=\frac{2\left(\varphi_{2}-\varphi_{1}\right)}{x_{2}-x_{1}}-d_{i}, i=0,1,2, \ldots, n \\
\varphi_{B}=\left[\frac{d_{i_{2}+1}-d_{i_{2}}}{2\left(x_{4}-x_{3}\right)}\right]\left(x_{B}-x_{3}\right)^{2}+d_{i_{2}}\left(x_{B}-x_{3}\right)+\varphi_{3} \\
\because d_{i_{2}}=0, d_{i_{2}+1}=\frac{2\left(\varphi_{4}-\varphi_{3}\right)}{x_{4}-x_{3}}-d_{i_{2}}, i_{2}=0,1,2, \ldots, n
\end{gathered}
$$

For natural spline, $d_{j}=0, d_{i}=0, d_{i_{2}}=0$ when $j=i=$ $i_{2}=0$. The unknown value of $\varphi_{A}$ is interpolated by using the known values of $\varphi_{1}$ and $\varphi_{2}$ whereas the unknown value of $\varphi_{B}$ is interpolated by using the known values of $\varphi_{3}$ and $\varphi_{4}$ at the $x$-axis. The details regarding COG method with biquadratic spline or $\mathrm{B}_{2}$-spline interpolation can be found in (Wee et. al., 2018; Wee et al., 2020)

\section{B. Implementation of Convolutional Perfectly Matched Layer}

The greatest challenges in the COG method is the solution of an interaction of EM wave with an unbounded region. An absorbing boundary condition (ABC) is applied to simulate an infinity lattice. In year 1994, the most robust and efficient technique proposed by Bérenger is by using the perfectly matched layer (PML) based ABC (Bérenger, 1994). The PML is used to truncate the OGG-FDTD lattice and act as an artificial lossy material that absorb signals. Nevertheless, the Bérenger split-field PML and the uniaxial PML are both ineffective at absorbing evanescent wave, where it suffers from late time reflections. According to Roden and Gedney the convolutional PML (CPML) able to solve the limitation of the traditional methods (Roden \& Gedney, 2000). Hence, this research was utilizing CPML technique to absorb both propagating and evanescent waves without reflections from the edge.

\section{FBTS Inversion Technique}

Forward-Backward Time Stepping (FBTS) is an inverse scattering technique with the goal of obtaining unknown electrical properties of an object by inverting scattered-field measurement data (Takenaka et al., 2015). The FBTS technique is used in this simulation work to detect breast cancer in two-dimensional. This technique has the ability to estimate the useful quantitative details of breast structure, such as electric properties, location and arbitrary shape. In this study, Gaussian pulse is chosen as the incident waveform and it is used as a source in the time domain. The Gaussian excitation is a sinusoidal wave with a Gaussian envelope function. Gaussian pulse plane wave provides a smooth roll off in frequency content and easy to implement provision for arbitrary incidence angle (Taflove \& Hagness, 2000).

As shown in Figure 2, the breast is encircled by 16 points source as antennas. Each of the 16 points was used as a transmitter antenna sequentially, while the remaining antennas ere used as receivers to absorb the scattered signals. The transmitter/receiver process was repeated until a measurement data set was obtained.

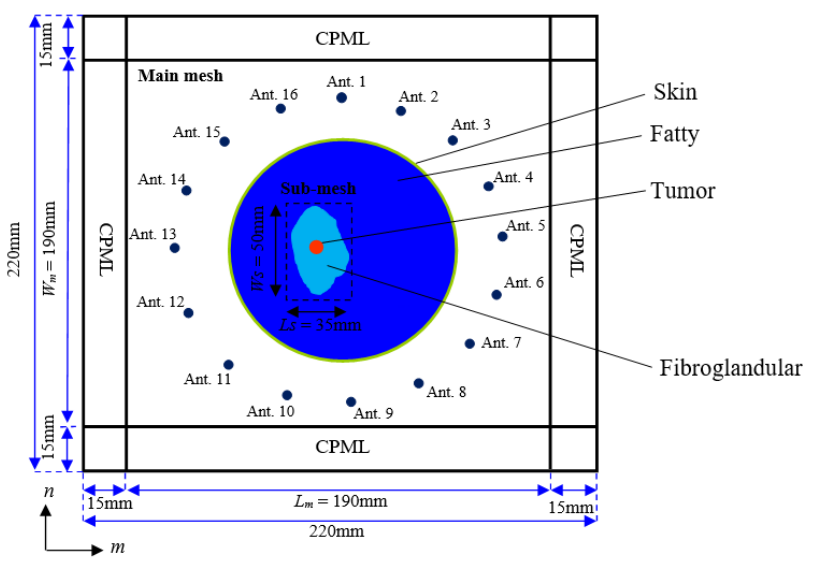

Figure 2. Configuration of homogeneous mostly fatty breast in $2 \mathrm{D}$ view

The forward step of the FBTS method begins with the excitation of Gaussian pulse signal to the estimated profile of the breast model. The COG method was used to determine the forward time-stepping scattered fields. The biquadratic spline interpolation was used in COG lattice to interpolate the EM field for the overlapping area between the main-mesh and the sub-mesh. Then, the forward time-stepping reconstructions data at the receiving point was collected and compared with the measurement data.

The COG method with biquadratic spline interpolation was used to measure the adjoint fields with time-stepping backward when the backward scattering. The biquadratic spline interpolation was used to interpolate the EM field between the main mesh and the sub-mesh in COG lattice for the overlapped region. Data on the time-stepping 
reconstructions were collected and compared at the receiving point.

This set of measurement data is compared to an equivalent simulation in which the same set of scattering data is measured for assumed electrical properties including relative permittivity $\left(\varepsilon_{r}\right)$ and conductivity $(\sigma)$ within the target objects. Since FBTS technique is an iterative technique, a costfunctional optimisation problem is formulated to minimise as Equation (2):

$Q(p)=\int_{0}^{T} \sum_{m=1}^{M} \sum_{n=1}^{N} K_{m n}(t)\left|v_{m}\left(p ; r_{n}^{r}, t\right)-\tilde{v}_{m}\left(r_{n}^{r}, t\right)\right|^{2} d t$

where $p$ is a set of electric parameter vector. $K_{m n}(t)$ is a nonnegative weighting function that takes value of zero at time $t$ $=T$, where $T$ is measurement time. The calculated EM field for the estimated medium parameter vector $p$ and the measured EM field for the $m^{t h}$ source are $v_{m}\left(p ; r_{n}^{r}, t\right)$ and $\tilde{v}_{m}\left(r_{n}^{r}, t\right)$, respectively.

Optimisation is the process of obtaining the most accurate result under given conditions (Rao, 2009). This technique can be classified into two types; constrained optimisation and unconstrained optimisation (Kelly, 1999). In this research, the cost function is utilising local minimisation approach in inverse scattering to measure the difference between the observed field and the computer for some trial parameters. The deterministic optimiser is integrated into FBTS technique which is conjugate gradient (CG) method. CG method is a class of unconstrained optimisation techniques and used to minimise the least-square error between calculated fields and the recorded output fields. There are several advantages of conjugate gradient method. Firstly, this method is superior, simple and easy to implement. It produces a faster convergence rate with low memory requirements. Secondly, the CG method requires a low memory and is very significant for solving a huge scale of nonlinear optimisation.

This research uses the Fletcher-Reeves (Fletcher \& Reeves, 1964) and Polak-Ribiere-Polyak (Polak \& Ribiere, 1969) method of conjugate gradient minimisation to solve the control of machine computations problems (Lasdon et al., 1967). It also used to minimise the cost functional $Q(p)$ with respect to the parameter vector $p$. The formulas for $\beta_{k}$, which are given below:
Fletcher-Reeves:

$$
\beta_{k}^{F R}=\frac{\left\|g_{k}\right\|^{2}}{\left\|g_{k-1}\right\|^{2}}
$$

Polak-Ribiere-Polyak:

$$
\beta_{k}^{P R P}=\frac{g_{k}\left(g_{k}-g_{k-1}\right)}{\left\|g_{k-1}\right\|^{2}}
$$

\section{RESULTS AND DISCUSSION}

According to Breast Imaging Reporting and Data System (BIRADS) (Silva \& Menotti, 2012), the mammograms for breast parenchymal density can be categorised into four classes: Class A (mostly fatty breast); Class B (scattered density breast); Class C (heterogeneously dense breast); and Class D (extremely dense breast). In order to analyse the accuracy of the COG method with biquadratic spline interpolation in FBTS to detect a malignancy tumour embedded in arbitrary shaped of the fibroglandular region, different classes of breast were described in Case A and Case B.

\section{A. Case A: Mostly Fatty Breast}

In this research, the mostly fatty breast was chosen in Case A as to validate the ability of the proposed method for breast cancer detection application. The mostly fatty breast is also known as Class A breast which is mostly fatty tissue contains few fibroglandular tissues. Hence, it is easier to spot abnormalities in the Class A breast than in Classes B, C and $\mathrm{D}$, respectively.

As shown in Figure 2, the FDTD lattice as the main mesh is consists of $190 \mathrm{~mm} \times 190 \mathrm{~mm}$ cells and surrounded by fifteencell of Convolution Perfectly Matched Layer (CPML). The sub-mesh overlaps with $35 \mathrm{~mm} \times 50 \mathrm{~mm}$ cells on top of the main-mesh. The cell size for main-mesh and sub-mesh is $\Delta x=1 \mathrm{~mm}$ and $\Delta y=1 \mathrm{~mm}$. The actual setting of numerical measured data is referred to nominal Debye parameters as shown in Table 1. Skin, adipose, fibroglandular, and tumour are the four kinds of breast tissues. The skin and adipose tissues were set as a circular shape with $50 \mathrm{~mm}$ and $48 \mathrm{~mm}$ radius, respectively. The thickness skin of the breast model is $2 \mathrm{~mm}$ in an imitation of a real human breast. A tumour with $4 \mathrm{~mm}$ radius was embedded within the arbitrary shape of the fibroglandular region. The skin and adipose regions were located at the centre of main mesh. Meanwhile, at the centre of the sub-mesh is the fibroglandular region and the tumour. 
Table 1. Electronic property parameters utilized for breast tissue (Johnson et al., 2009)

\begin{tabular}{ccccc}
\hline Layer & Tissue & Size & $\begin{array}{c}\boldsymbol{\varepsilon}_{\mathbf{r}} \\
(\mathbf{F} / \mathbf{m})\end{array}$ & $\begin{array}{c}\boldsymbol{\sigma} \\
(\mathbf{S} / \mathbf{m})\end{array}$ \\
\hline $\begin{array}{c}\text { Layer 1 } \\
\text { (Main }\end{array}$ & Skin & $50 \mathrm{~mm}$ & 36.73 & 1.43 \\
mesh) & Fat & $\begin{array}{c}48 \mathrm{~mm} \\
\text { (Radius) }\end{array}$ & 9.98 & 0.18 \\
& & (Radius) & & \\
Layer 2 & Fibroglandular & - & 21.45 & 0.46 \\
$\begin{array}{c}\text { (Sub- } \\
\text { mesh) }\end{array}$ & Tumour & 4 mm & 53.62 & 1.19 \\
& & (Radius) & \\
\hline
\end{tabular}

The breast is surrounded by 16 points source as antennas. The antennas are arranged in a circular shape with a radius of $85 \mathrm{~mm}$. Each of the 16 points was used sequentially as a transmitter antenna while the remaining antennas were utilised as receivers to collect the scattered signals. The transmitter/receiver process was repeated until a set of transmit/receive data was obtained. A sinusoidal modulated Gaussian pulse acted as an excitation signal with a centre frequency of $2.0 \mathrm{GHz}$ and a bandwidth of $1.3 \mathrm{GHz}$. The proposed method was simulated up to 100 iterations in order to reconstruct the image of internal breast composition.

The actual profiles of relative permittivity and conductivity for the mostly fatty breast are illustrated in Figure 3. Figure 4 shows the reconstructed breast profiles utilising the FDTD method in FBTS. The tumour embedded in the fibroglandular region was detected and reconstructed. However, the FDTD method in FBTS has a limitation on reconstruction of the arbitrary shape of the fibroglandular region. Thus, the COG method with biquadratic spline interpolation in FBTS was proposed in this research in order to improve the quality of the reconstructed images. As shown in Figure 5, the proposed method successfully reconstructed the internal composition of mostly fatty breast. The location, size, and shape of the tumour were also determined.

The accuracy of the proposed numerical method was then investigated by using the Mean Square Error (MSE). The MSE of the reconstructed electrical properties for FDTD method in FBTS are $\mathbf{1 7 . 0 9}$ for relative permittivity and $5.22 \times 10^{-2}$ for conductivity. The MSE for proposed method is lower than FDTD method in FBTS with 12.65 for relative permittivity, and $3.02 \times 10^{-2}$ for conductivity.

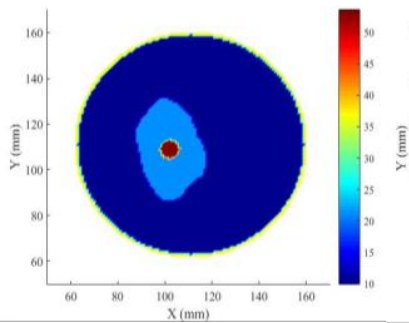

(a) Actual relative permittivity

Figure 3. Actual dielectric profiles of Case A

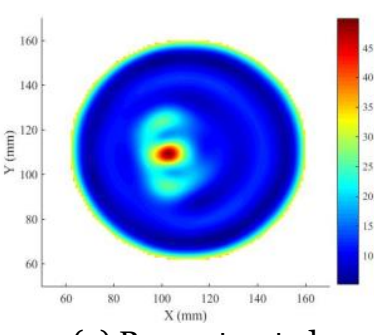

(a) Reconstructed relative permittivity

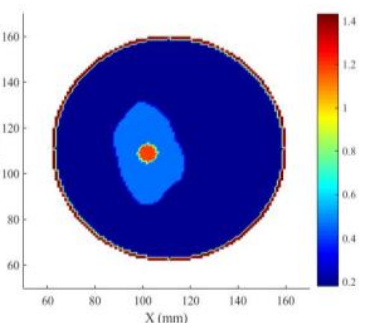

(b) Actual conductivity

Figure 4. Reconstructed dielectric profiles of Case A for

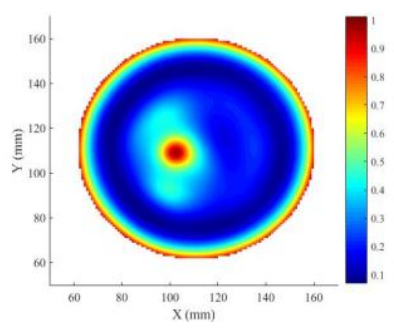

(b) Reconstructed conductivity
FDTD method in FBTS

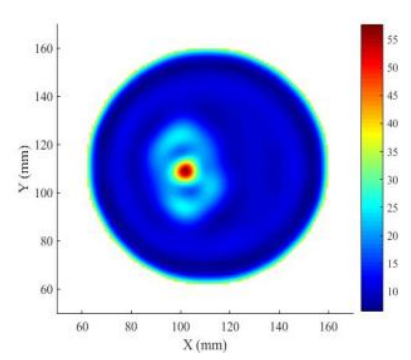

(a) Reconstructed relative permittivity
Figure 5. Reconstructed dielectric profiles of Case A for OGG-FDTD method with biquadratic spline interpolation in FBTS

Figure 6 shows a cross-sectional view of actual and reconstructed profiles for Case A. The solid line represents the actual profile, while the dashed line signifies the reconstructed profile for FDTD method in FBTS, and the dashdot line indicates the reconstructed profile for COG method with biquadratic spline interpolation in FBTS. The high contrast of dielectric properties between malignant and normal breast tissues can be clearly seen in this figure. The peak values of tumour for the proposed method are more similar to the actual values of $\varepsilon_{\max }=53.62 \mathrm{~F} / \mathrm{m}$ and $\sigma_{\max }=$ $1.19 \mathrm{~S} / \mathrm{m}$ as compared to the FDTD method in FBTS. 


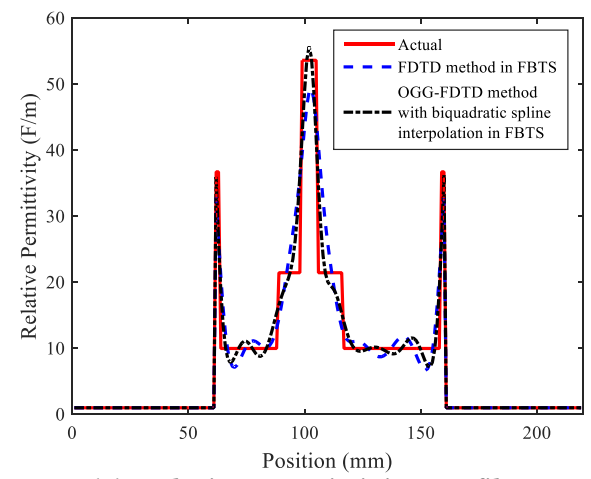

(a) Relative permittivity profile

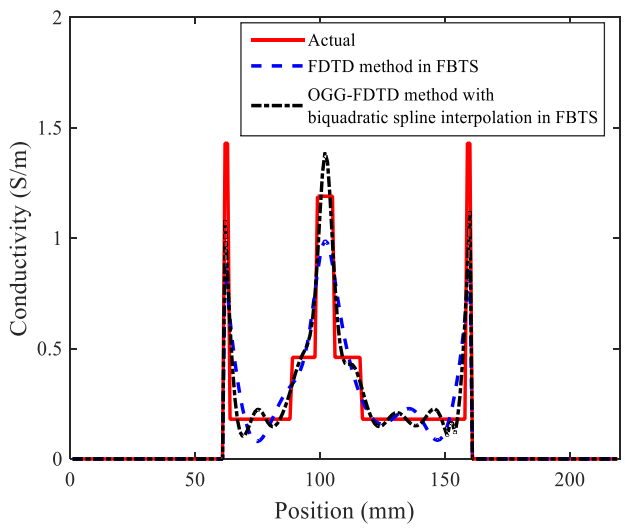

(b) Conductivity profile

Figure 6. Cross-sectional view of actual and reconstructed dielectric profiles along $\mathrm{x}$-axis at $\mathrm{y}=110$ for Case $\mathrm{A}$

The normalised functional error versus number of iterations is illustrated in Figure 7. As the number of iterations increased, the normalised functional error decreased. It also found that the values of normalised functional error for the proposed method are lower compared to the FDTD method in FBTS. The result indicated that the OGG-FDTD method in FBTS can accurately reconstruct breast model as compared to the FDTD method in FBTS.

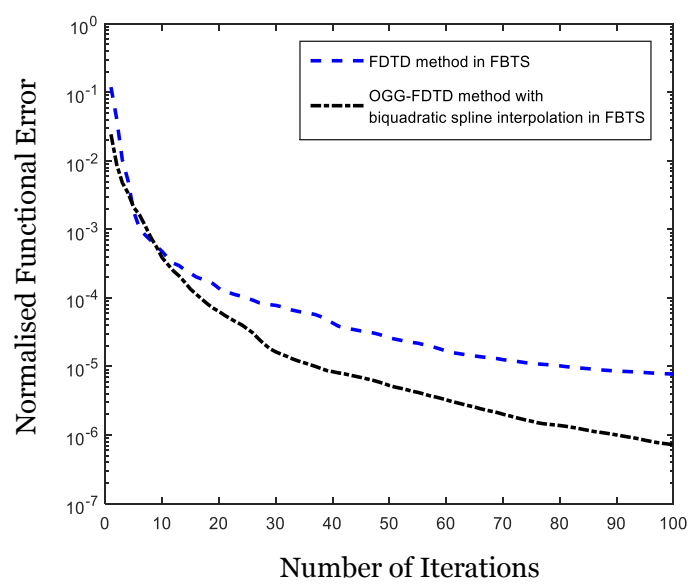

Figure 7. Normalised functional error versus number of iterations for Case A

\section{B. Case B: Extremely Dense Breast}

In this research, the extremely dense breast was chosen in Case B as to evaluate the proposed method. The extremely dense breast (also known as Class D breast) contains considerably more fibrous and glandular tissue compared to other classes. The sensitivity of mammography for this type of breast is lower. Therefore, it is difficult to detect cancer in a Class D breast. The main-mesh was set to $190 \mathrm{~mm} \times 190 \mathrm{~mm}$ grids. The sub-mesh was set to $50 \mathrm{~mm} \times 50 \mathrm{~mm}$ grids and it was modelled as an arbitrary shape fibroglandular region. A tumour of $4.0 \mathrm{~mm}$ radius was added in the fibroglandular region of the numerical breast. The other parameter settings of Case B were set to be similar to those of Case A.

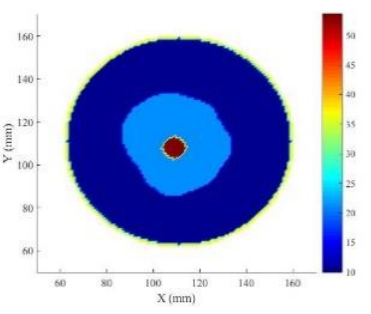

(a) Actual relative permittivity

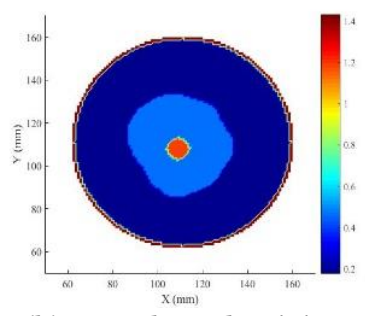

(b) Actual conductivity
Figure 8. Actual dielectric profiles of Case B

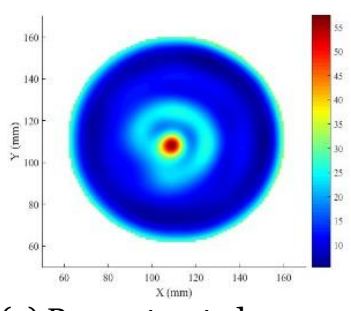

(a) Reconstructed relative permittivity

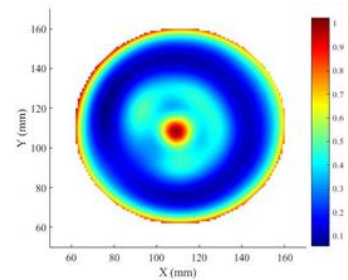

(b) Reconstructed conductivity
Figure 9. Reconstructed dielectric profiles of Case B for FDTD method in FBTS

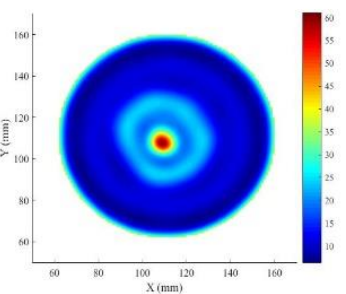

(a) Reconstructed relative permittivity

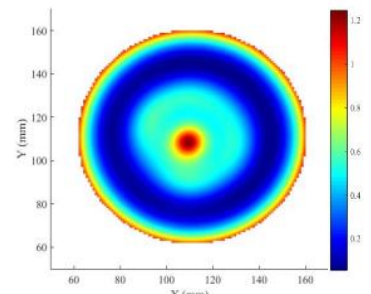

(b) Reconstructed conductivity
Figure 10. Reconstructed dielectric profiles of Case B for OGG-FDTD method with biquadratic spline interpolation in FBTS 
Figure 8 shows the actual profiles of relative permittivity and conductivity for an extremely dense breast in Case B. Figure 9 shows the reconstructed profiles of an extremely dense breast utilising the FDTD method in FBTS. As can be seen, the FDTD method in FBTS has difficulties in modelling the curved boundaries and small features for arbitrary shape of fibroglandular region due to its restriction to inherent orthogonal grids. Therefore, the OGG-FDTD method with biquadratic spline interpolation in FBTS was proposed in this research to improve the efficiency of the FDTD method in FBTS as shown in Figure 10. The results obtained show that the proposed method has the ability to detect a malignant tumour embedded in arbitrary shape of the fibroglandular region. The proposed method successfully reconstructed the quantification of space in finite-sized cells for an arbitrary shape of the fibroglandular region.

Figure 11 shows a cross-sectional view of actual and reconstructed profiles for Case B. The solid line represents the actual profile, the dashed line denotes the reconstructed profile for FDTD method in FBTS, and the dash-dot line indicates the reconstructed profile for the proposed method. The peak values of tumour for FDTD method in FBTS are $\varepsilon_{\max }=53.51 \mathrm{~F} / \mathrm{m}$ and $\sigma_{\max }=1.096 \mathrm{~S} / \mathrm{m}$ while the proposed method shows the results of $\varepsilon_{\max }=62.06 \mathrm{~F} / \mathrm{m}$ and $\sigma_{\max }=$ $1.248 \mathrm{~S} / \mathrm{m}$. Hence, it has been proven that the proposed method can determine the dielectric profiles accurately as compared to the FDTD method in FBTS.

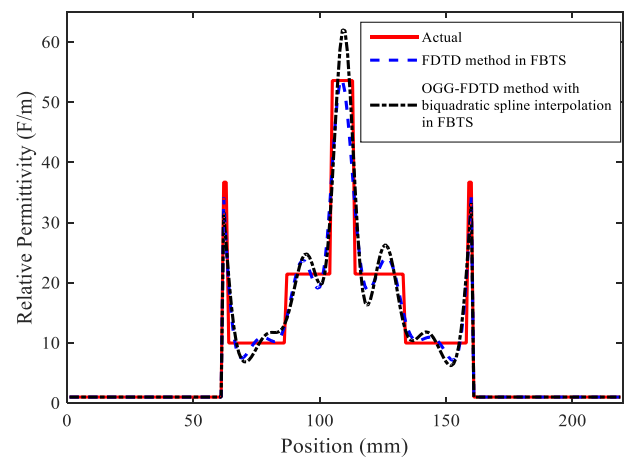

(a) Relative permittivity profile

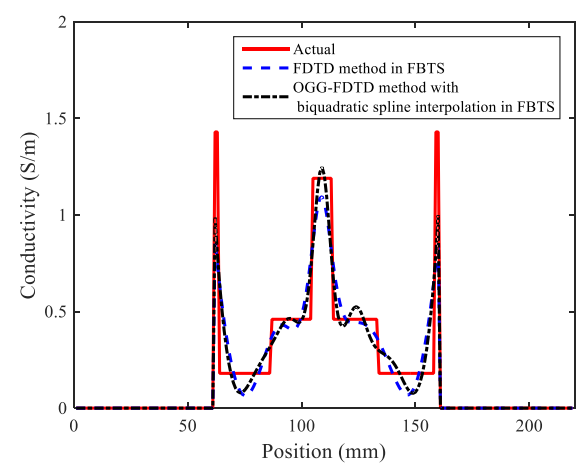

(b) Conductivity profile

Figure 11. Cross-sectional view of actual and reconstructed dielectric profiles along $\mathrm{x}$-axis at $\mathrm{y}=110$ for Case $\mathrm{B}$

In order to analyse the efficiency of the proposed method, the reconstructed profiles for the breast were evaluated by using Mean Square Error (MSE) and normalised functional error. It was found that the MSE and normalised functional error for the COG method with biquadratic spline interpolation in FBTS achieved significantly lower values than the FDTD method in FBTS as shown in Table 2 and Figure 12.

Table 2. Mean Square Error (MSE) of dielectric profiles for Case B

\begin{tabular}{lcc}
\hline \multirow{2}{*}{ Methods } & \multicolumn{2}{c}{ MSE } \\
\cline { 2 - 3 } & $\begin{array}{c}\text { Relative } \\
\text { Permittivity } \\
\text { Profile }\end{array}$ & $\begin{array}{c}\text { Conductivity } \\
\text { Profile }\end{array}$ \\
& 16.51 & $6.84 \times 10^{-2}$ \\
\hline FDTD method in & & \\
FBTS & & \\
COG method with & & \\
biquadratic spline \\
interpolation in \\
FBTS
\end{tabular}




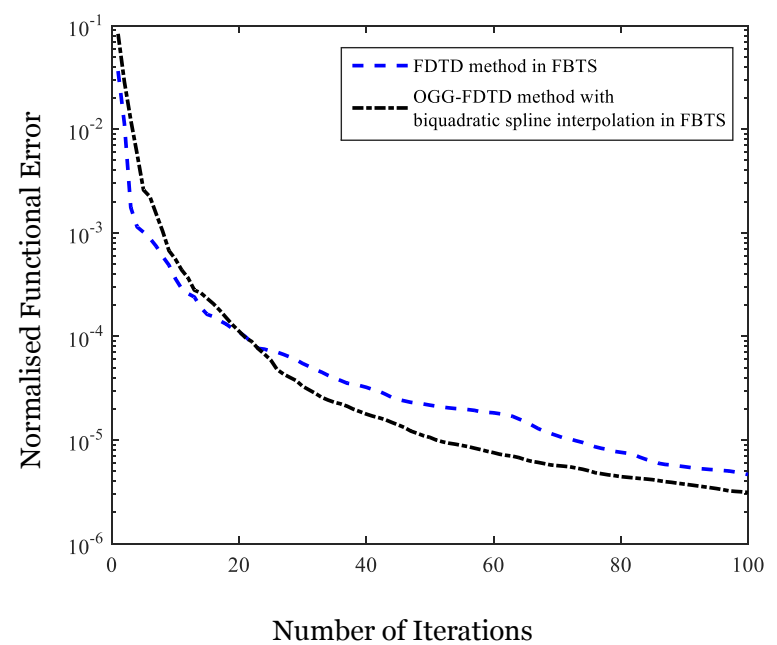

Figure 12. Normalised functional error versus number of iterations for Case B

The numerical results showed that the proposed method able to solve the drawback for FDTD method that is difficult to exactly generate meshes for electromagnetic structures with curved boundaries and small features due to its restriction to inherent orthogonal grids. The results showed that the proposed method provided better reconstructed images and accurately retrieved the dielectric profiles of the investigation area as compared to the FDTD method in FBTS. The details regarding COG method with biquadratic spline or $B_{2}$-spline interpolation for arbitrary shaped objects and inhomogeneous actual imaging reconstruction can also be found in (Wee et al., 2020).

\section{CONCLUSION}

The COG method with biquadratic spline interpolation in FBTS inverse scattering technique was used to reconstruct the breast composition. The homogeneous mostly fatty breast and extremely dense breast were used to evaluate the proposed method. The numerical results indicated that the proposed method was able to obtain the useful quantitative information details of the breast structure such as shape, dimension, location and dielectric properties. Reconstructed images indicated clearly the position and size of a tumour placed within an arbitrary shape of the fibroglandular region. Hence, it can be stated that the proposed method can further improve the quality of reconstructed images.

\section{ACKNOWLEDGEMENT}

This research was supported by Dana Pelajar PhD (DPP) grant scheme (Fo2/DPP/1602/2017). The authors fully acknowledged the Ministry of High Education Malaysia (MOHE) and Universiti Malaysia Sarawak (UNIMAS) for the approved fund which makes this important research viable and effective.

\section{REFERENCES}

Aldhaeebi, MA, Alzoubi, K, Almoneef, TS, Bamatraf, SM, Attia, H \& M Ramahi, O 2020, 'Review of microwaves techniques for breast cancer detection', Sensors, vol. 20, no. 8, pp. 2390.

American Cancer Society 2019, Cancer facts \& figures 2019, viewed 23 December 2020, $<$ https://www.cancer.org/latest-news/facts-and-figures2019.html>.

Berenger, JP 1994, 'A perfectly matched layer for the absorption of electromagnetic waves', Journal of Computational Physics, vol. 114, no. 2, pp. 185-200.

Brennan, M \& Houssami, N 2016, 'Discussing the benefits and harms of screening mammography', Maturitas, vol. 92, pp. $150-153$
Fletcher, R \& Reeves, CM 1964, 'Function minimization by conjugate gradients', The Computer Journal, vol. 7, no. 2, pp. 149-154.

Gao, F, Van Veen, BD, \& Hagness, SC 2015, 'Sensitivity of the distorted born iterative method to the initial guess in microwave breast imaging', IEEE Transactions on Antennas and Propagation, vol. 63, no. 8, pp. 3540-3547.

Hang, JA, Sim, L \& Zakaria, Z 2017, 'Non-invasive breast cancer assessment using magnetic induction spectroscopy technique', International Journal of Integrated Engineering, vol. 9, no. 2, pp. 54-6o.

Jiménez-Mejía, E \& Herrera-Murcia, J 2015, 'Validation of a non-uniform meshing algorithm for the $3 \mathrm{D}$-FDTD method 
by means of a two-wire crosstalk experimental set-up,' Ingeniería e Investigación, vol. 35, pp. 98-103.

Johnson, JE, Takenaka, T, Ping, KAH, Honda, S \& Tanaka, T 2009, 'Advances in the 3-D Forward-Backward TimeStepping (FBTS) inverse scattering technique for breast cancer detection', IEEE Transactions on Biomedical Engineering, vol. 56, no. 9, pp. 2232-2243.

Kelley, CT 1999, 'Iterative methods for optimization', Philadelphia, Pennsylvania: Society for Industrial and Applied Mathematics.

Lasdon, L, Mitter, S \& Waren, A 1967, 'The conjugate gradient method for optimal control problems', IEEE Transactions on Automatic Control, vol. 12, no. 2, pp. 132-138.

Li, Y, Porter, E, Santorelli, A, Popović, M, \& Coates, M 2017, 'Microwave breast cancer detection via cost-sensitive ensemble classifiers: phantom and patient investigation', Biomedical Signal Processing and Control, vol. 31, pp. 366376.

Mashal, A, Sitharaman, B, Li, X, Avti, PK, Sahakian, AV, Booske, JH, \& Hagness, SC 2010, 'Toward carbonnanotube-based theranostic agents for microwave detection and treatment of breast cancer: Enhanced dielectric and heating response of tissue-mimicking materials', Biomedical Engineering, IEEE Transactions on, vol. 57, no. 8, pp. 1831-1834.

Neira, LM, Van Veen, BD \& Hagness, SC 2017, 'Highresolution microwave breast imaging using a $3-\mathrm{D}$ inverse scattering algorithm with a variable-strength spatial prior constraint', IEEE Transactions on Antennas and Propagation, vol. 65, no. 11, pp. 6002-6014.

Nilavalan, R, Craddock, IJ \& Railton, CJ 2002, 'Quantifying numerical dispersion in non-orthogonal FDTD meshes', IEE Proceedings-Microwaves, Antennas and Propagation, vol. 149, no. 1, pp. 23-27.

Polak, E \& Ribiere, G 1969, 'Note Sur la convergence de directions conjug `ees, Rev. Francaise Informat Recherche Operationelle', Mathematical Modelling and Numerical Analysis-Modélisation Mathématique et Analyse Numériqu', vol. 3, no. R1, pp. 35-43.

Radiological Society of North America Inc. 2019, Mammography, viewed 2 July 2019, <https://www. radiologyinfo.org/en/info.cfm?pg=mammo $>$.

Rao, SS 2009, Engineering optimization: Theory and practice, John Wiley \& Sons, New Jersey, United State.

Roden, JA \& Gedney, SD 2000, 'Convolutional PML (CPML): An efficient FDTD implementation of the CFS-PML for arbitrary media,' Microwave and Optical Technology Letters, vol. 27, no. 5, pp. 334-338.

Schneide, JB 2016, 'Understanding the finite-difference timedomain method', $\mathrm{PhD}$ thesis, School of Electrical Engineering and Computer Science, Washington State University, Washington, US.

Siegel, RL, Miller, KD \& Jemal, A 2016, 'Cancer statistics, 2019', A Cancer Journal for Clinicians, vol. 69, pp. 7-34.

Silva, WR \& Menotti, D 2012, 'Classification of mammograms by the breast composition', in Proceedings of the International Conference on Image Processing, Computer Vision, and Pattern Recognition (IPCV).

Taflove, A \& Hagness, SC 2000, Computational electrodynamics, London: Artech house publishers.

Takenaka, T, Jia, H \& Tanaka, T 2015, 'Microwave imaging of electrical property distributions by a Foward-Backward Time-Stepping method', Journal of Electromagnetic Waves and Applications, vol. 14, pp. 1609-1626.

Takenaka, T, Moriyama, T, Ping, KAH \& Yamasaki, T 2010, 'Microwave breast imaging by the filtered forwardbackward time-stepping method', in Proceedings of the URSI International Symposium on Electromagnetic Theory Tartar, M, Comstock, CE \& Kipper, MS 2008, Breast cancer imaging: a multidisciplinary, multimodality approach: Philadelphia, Elsevier Health Sciences, US.

Wang, L 2019, 'Multi-frequency holographic microwave imaging for breast lesion detection,' IEEE Access, vol. 7, pp. 83984-83993.

Wee, BS, Sahrani, S \& Ping, KAH 2018, 'B2-spline interpolation technique for overset grid generation and Finite-Difference Time-Domain method,' Progress In Electromagnetics Research C, vol. 86, pp. 177-190.

Wee BS, Ping K A H \& Sahrani S 2020, 'Arbitrary shaped objects detection and reconstruction through Overset Grid Generation Method with $\mathrm{B}_{2}$-spline interpolation in Forward-Backward Time-Stepping inverse scattering', Applied Computational Electromagnetics Society Journal, vol. 35, no. 3, pp. 295-304.

Winters, DW, Shea, JD, Kosmas, P, Van Veen, BD \& Hagness, SC 2009, "Three-dimensional microwave breast imaging: Dispersive dielectric properties estimation using patientspecific basis functions', IEEE Transactions on Medical Imaging, vol. 28, no. 7, pp. 969-981.

Yee, KS 1966, 'Numerical solution of initial boundary value problems involving Maxwell's equations in isotropic media', IEEE Transactions on Antennas and Propagation, vol. 14, no. 3, pp. 302-307. 